\title{
Lupus: The Essential Clinician's Guide (2nd Edition)
}

Daniel J. Wallace. Oxford: Oxford University Press, 2014, 128 pages, $\$ 35$ US

Dr. Daniel Wallace has published widely on the topic of systemic lupus erythematosus (SLE), most importantly as author and editor of the definitive reference for SLE, Dubois' Lupus Erythematosus and Related Syndromes. His most recent offering, Lupus: The Essential Clinician's Guide, 2nd ed., serves as a useful rapid reference, taking about 100 pages to discuss a wide range of topics related to this complex disease.

The publication is organized into 14 chapters touching on pathogenesis, classification criteria for SLE and related disease, interpretation of immunologic investigations, clinical manifestations of disease, treatment, and emerging therapies. Numerous tables and diagrams populate each chapter, key for a quick review. Clinicians will particularly appreciate a chapter focused on treatment, organized by organ system involvement with specific therapies suggested for each manifestation. Treatment algorithms and a guide to topical steroid treatments for management of cutaneous disease are other clinically useful highlights. Chapters explaining classification of SLE, similar disorders, and outcome measures, are important resources for clinicians, ensuring accurate diagnosis and assessment in daily clinical evaluation.

Overall, Dr. Wallace succeeds at using this succinct text to distill key clinical facts and offer numerous pearls for busy clinicians to aid in the management of patients with SLE. I recommend this text for any rheumatology trainee or practicing rheumatologist as a handy daily reference for all aspects of SLE care.

MARK MATSOS, MD, Department of Medicine, McMaster University, 25-708 Charlaton Ave. East, Hamilton, Ontario L8N 1Y2,

Canada.E-mail: markmatsos@gmail.com

J Rheumatol 2014;41:11; doi:10.3899/jrheum.140826 